POS $\quad$ PROCEEDINGS

\title{
Design of a Prototype EDM Storage Ring
}

\author{
Andreas Lehrach \\ Forschungszentrum Jülich, Institut für Kernphysik (IKP-4) \\ Wilhelm-Johnen-Str., 52428 Jülich, Germany \\ JARA-FAME und III. Physikalisches Institut B, RWTH Aachen University \\ Otto-Blumenthal-Str., 52074 Aachen, Germany \\ E-mail: a.lehrach@fz-juelich.de
}

\section{Siegfried Martin*}

Forschungszentrum Jülich, Institut für Kernphysik (IKP-4)

Wilhelm-Johnen-Str., 52428 Jülich, Germany

E-mail: s.martinefz-juelich.de

\section{Richard M. Talman}

Cornell University, Laboratory for Elementary-Particle Physics (LEPP)

245 East Avenue, Ithaca, NY 14853-2501, USA

E-mail: richard.talman@cornell.edu

\section{for the Charged Particle Electric Dipole Moment (CPEDM) Collaboration ${ }^{\dagger}$}

This paper summarizes the efforts to design a prototype EDM (Electric Dipole Moment) storage ring in the frame of the CPEDM collaboration, with predominantly electric bending. Operated at proton beam energies between 30 and $50 \mathrm{MeV}$, the main purpose of this ring will be to carry out R\&D work related to a final $233 \mathrm{MeV}$ frozen-spin proton EDM ring. After demonstrating satisfactory beam lifetime and spin coherence time in the electrostatic ring, clockwise and counterclockwise beam operation, beam spin control, beam-based element alignment, and methods for reducing systematic errors in EDM measurements will be investigated. At these reduced proton beam energies, the (weak) superimposed magnetic field required to freeze the proton spins can be provided by powering the iron-free (hysteresis-free) "cosine-theta" coils built into the ring design. This will allow the development of EDM measurement techniques and permit the first direct precision measurement of the proton EDM.

23rd International Spin Physics Symposium - SPIN2018 -

10-14 September, 2018

Ferrara, Italy

\footnotetext{
*Speaker.

${ }^{\dagger}$ http://pbc.web.cern.ch/edm/edm-default.htm.
} 


\section{Introduction}

Permanent EDMs of fundamental particles violate both time invariance $\mathrm{T}$ and parity P. Assuming the CPT theorem this implies CP violation. The Standard Model predicts non-vanishing EDMs, their magnitudes, however, are expected to be unobservably small with current techniques. Hence, the discovery of a non-zero EDM would be a signal for "new physics".

Up to now most of the EDM measurements were done with neutral particles. The best current EDM limit for the neutron is presently $3 \cdot 10^{-26} \mathrm{e}-\mathrm{cm}[1]$. With new techniques, it is now possible to perform dedicated EDM experiments with charged hadrons at storage rings where polarized beams are available. If an EDM exists, the spin vector will experience an additional torque in the electromagnetic fields of the storage ring resulting in a change of the original spin direction. If the bending fields in a storage ring are adjusted according to the particle momentum in such a way that the longitudinally polarized spins of the particle beam are kept aligned ("frozen") with their momenta, electric fields in the rest frame of the particles will precess the spin via the EDM into the vertical direction. This change of the vertical component of the beam polarization from early to late storage times is the signature of the EDM signal [2], which can be determined with the help of a polarimeter.

The ultimate goal of the CP EDM collaboration is to design, build, and operate a proton all-electric ring with frozen spins at the magic momentum of about $0.7 \mathrm{GeV} / \mathrm{c}(233 \mathrm{MeV})$. For this purpose a roughly $500 \mathrm{~m}$ long storage ring is required, capable to inject and simultaneously store counterrotating beams to reach a desired sensitivity for the EDM measurement in the order of $10^{-29} \mathrm{e}-\mathrm{cm}$ [3]. A step-wise approach is pursued, starting with ongoing technical feasibility studies at the Cooler Synchrotron COSY in Jülich, followed by a first direct EDM measurement of a charged particle at COSY [5]. On a longer time scale, it is foreseen to construct an intermediate prototype proton EDM ring, and finally design and build the high precision proton EDM storage ring [4].

The present paper describes a starting point lattice for the prototype EDM ring in terms of geometry, type and strength of the elements. The storage ring should be small and simple, and as inexpensive as possible.

\section{Prototype EDM Ring Layout}

\subsection{Design Requirements and Considerations}

Before designing a final EDM ring with ultimate precision, a smaller-scale prototype ring (PT EDM ring) must be constructed first to prove critical features and capabilities required for EDM measurements. The proposed ring is designed to be capable of achieving its claimed goals:

- Storage of high intensity beams for a sufficiently long time,

- Beam injection with multiple polarization states (longitudinal and sideways) in both clockwise (CW) and counter-clockwise (CCW) beams,

- Simultaneously circulating CW and CCW beams with frozen spin capability,

- Magnetic shielding to reduce the ambient radial magnetic field components, 
- Polarization measurements for both $\mathrm{CW}$ and $\mathrm{CCW}$ beams using the same target,

- Phase-space beam cooling (electron cooling) before injection, or stochastic cooling.

Spin tracking calculations are needed to verify the level of precision needed in the ring construction and the handling of systematic errors. For a detailed study during beam storage and buildup of the EDM signal, one needs to track a large sample of particles for billions of turns. The COSYInfinity [6] and Bmad [7] simulation programs are utilized for this purpose. Given the complexity of the tasks, particle and spin tracking programs have been benchmarked and simulation results compared to beam and spin experiments at COSY to ensure the required accuracy of the results [8].

When constructed, the proton EDM experiment will be the largest electrostatic ring ever built. It will have unique features, such as counter-rotating beams and strenuous alignment and stability requirements. It may also require stochastic cooling and weak magnetic focusing consistent with dual beam operation. Parameter specifications have to be defined carefully to achieve the given EDM precision, and discussion of measures to be taken to improve this precision by refining the parameter tolerances and perfecting the methods of systematic error reduction. The prototype ring should provide empirical experience needed to assess the systematic EDM errors.

The PT EDM ring will have a circumference of about $100 \mathrm{~m}$ and will be operated in two modes. The first mode would work with all-electric bending (at $30 \mathrm{MeV}$ proton energy). This would show that such a concept works and the feasibility of the ring with simultaneous counter-rotation beams. The second mode would expand the operating range of proton energies to $45 \mathrm{MeV}$ with the addition of magnetic bends (air core). With this combination, "frozen" spin operation could be demonstrated for a proton beam, other spin manipulation tools developed, and a reduced-precision proton EDM value measured. Alternating fills in counter-rotating directions would allow cancellation of the average radial magnetic field that is the leading cause of systematic error. The systematic error will strongly be associated with the needed magnetic field reversal.

\subsection{Ring Design}

This section describes a starting-point lattice for the PT EDM ring. The present ring design for the prototype is a fourfold symmetry "squared" ring with $8 \mathrm{~m}$ long straight sections as shown in Fig. 1. This is the result of studies discussing different lattices like round and racetrack type shapes. The basic beam parameters are given in Table 1.

The lattice consists of 4 unit cells each of them bending $90^{\circ}$ in the arcs. Each of them compromises the following focusing structure: QF - B - QD - B - QF, with QF is a focusing quadrupole, QD a defocusing quadrupole and B an electric/magnetic bending unit. The bend elements consist of electric and magnetic bending. The pure electric bending provides to required rigidity for $30 \mathrm{MeV}$ protons. For a proton energy of $45 \mathrm{MeV}$ magnetic bending has to be applied in addition. The straight sections must house two injection regions for clockwise (CW) and counter-clockwise $(\mathrm{CCW})$ operation. There will as well be a quadrupole QSS in the center of each of the straight 


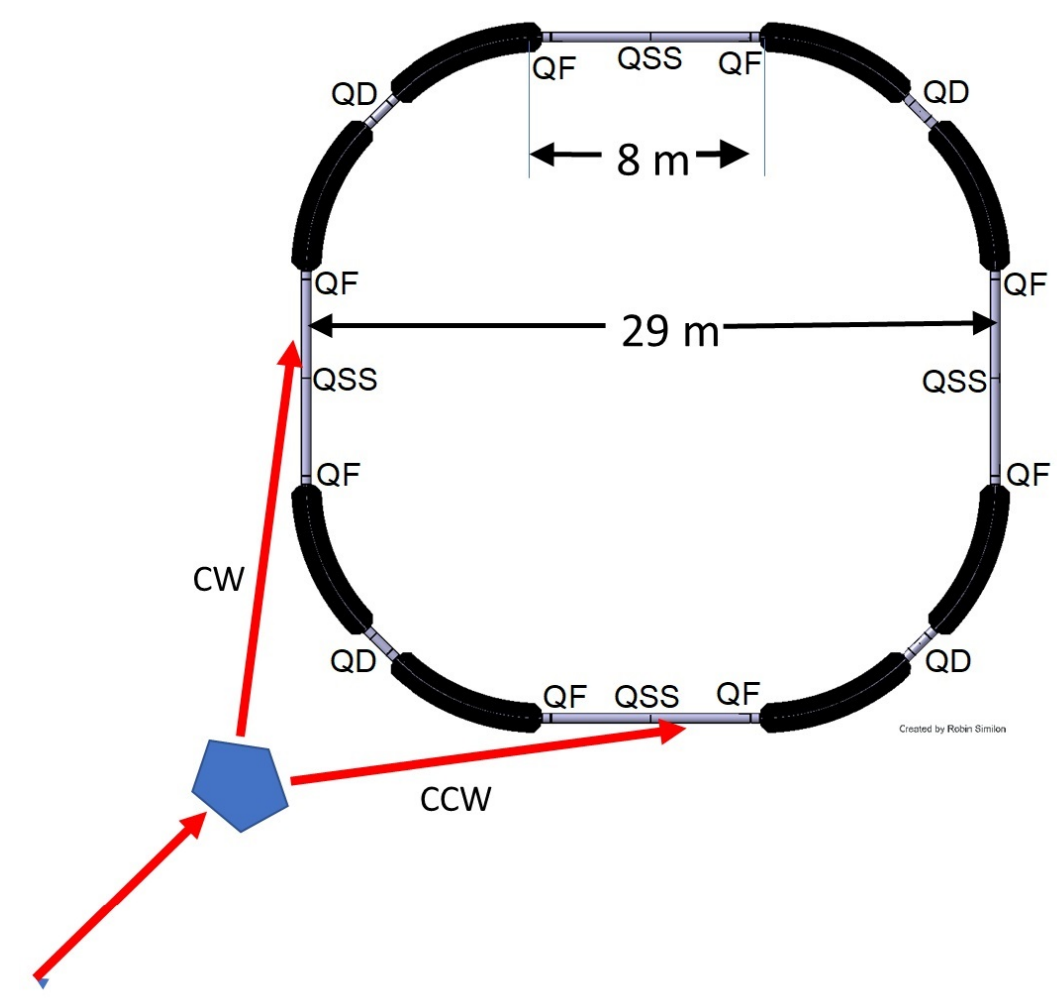

Figure 1: The basic layout of the PT EDM ring, consisting of eight dual, superimposed electric and magnetic bends; three families of quadrupoles (focusing: QF, defocusing: QD, and straight section: QSS); and four $8 \mathrm{~m}$ long straight sections. The total circumference is about $100 \mathrm{~m}$. Injection lines for injecting countercirculating beam are represented just as stubs.

section to gain additional tuning possibilities. The lattice is designed to allow a variable horizontal tune $Q_{x}$ between 1 and 2 and a vertical tune $Q_{y}$ between 1.6 and 0.1 in the vertical plane (see Fig. 2). The flexibility of the lattice is one of the goals for the design. The betatron tunes can be varied over a large range as shown in Figs. 2 and 3. This way, the lattice can be adjusted for ultra-weak to moderate focusing.

This tuning capability enables us to adjust the ring for either larger beam acceptance resulting in long beam lifetime, if strong vertical focusing is applied or reduced radial magnetic fields, if ultra-weak vertical focusing is adjusted. Typical values of the beta functions is given in figure 4 .

The performance of the PT EDM ring has to be discussed in terms of systematic errors, beam lifetime, spin coherence time, instabilities and beam dynamic limits like intrabeam scattering, multiple rest gas scattering, and wake field effects. The basic parameters for the PT EDM ring elements are given in Tables 2 and 3.

Beam injection: Injection into the PT EDM ring will closely resemble injection into a nominal all-electric ring. In particular there will be an even number of bunches in each beam, with alternating sign polarizations, whether in single beam or counter-circulating beam operation. The injector for the PT EDM ring could be the electron-cooled beam from COSY or make use of equip- 
Table 1: Basic beam parameters.

\begin{tabular}{lccc}
\hline & $E$ only & $E \times B$ & unit \\
\hline kinetic energy & 30 & 45 & $\mathrm{MeV}$ \\
$\beta=v / c$ & 0.247 & 0.299 & \\
momentum & 239 & 294 & $\mathrm{MeV} / \mathrm{c}$ \\
magnetic rigidity $B \rho$ & 0.798 & 0.981 & $\mathrm{~T} \cdot \mathrm{m}$ \\
electric rigidity $E \rho$ & 59.071 & 87.941 & $\mathrm{MV}$ \\
$\gamma$ (kinetic) & 1.032 & 1.048 & \\
emittance $\varepsilon_{x}=\varepsilon_{y}$ & 1.0 & 1.0 & $\mathrm{~mm} \cdot \mathrm{mrad}$ \\
acceptance $a_{x}=a_{y}$ & 1.0 & 10.0 & $\mathrm{~mm} \cdot \mathrm{mrad}$ \\
\hline
\end{tabular}

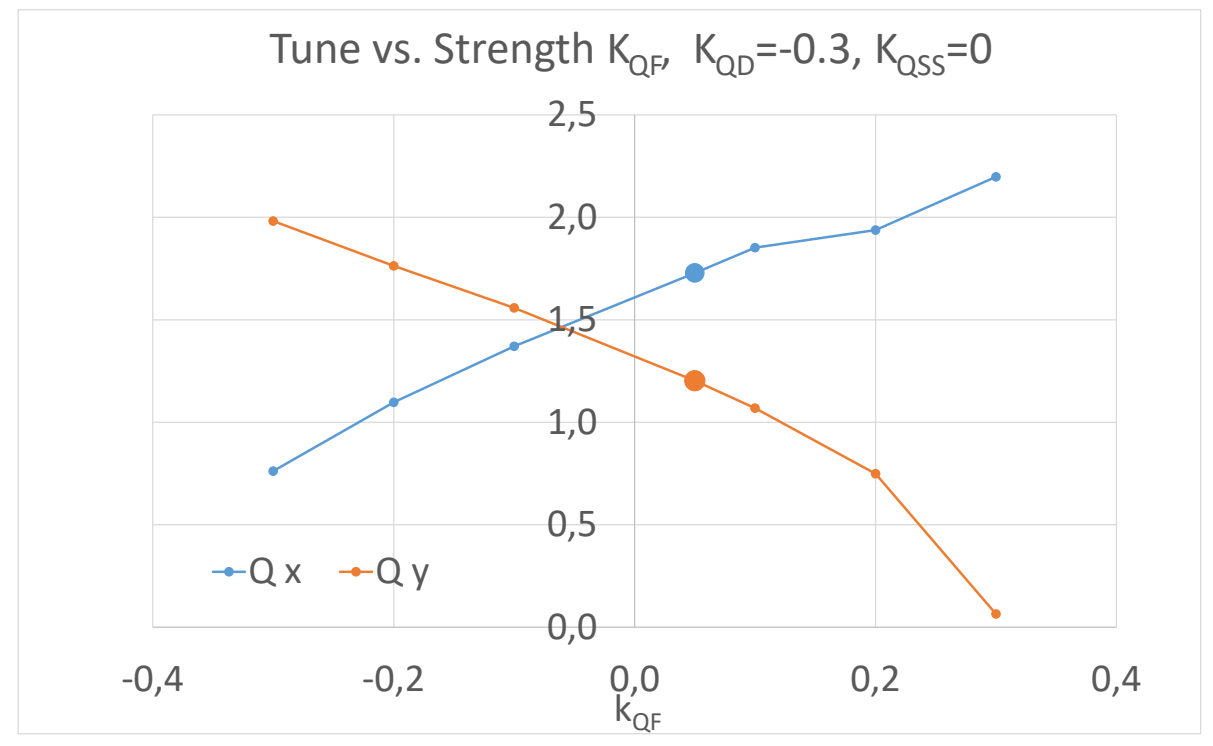

Figure 2: Betatron tune settings without applying the quadrupoles in the straight sections QSS. The horizontal betatron tune $Q_{x}$ and vertical betatron tune $Q_{y}$ are plotted versus the strength of the horizontally focusing quadrupole family QF in the arcs. The horizontally defocusing quadrupole families QD in the arcs and quadrupoles in the straight section QSS are constant. The marked points are continued in Fig. 3

ment at CERN. The beams will be protons in the 30 to $45 \mathrm{MeV}$ range, in a cooled phase space of $1 \pi \mathrm{mm} \cdot \mathrm{mrad}$, with the beams bunched into $2,4,6$ or 8 bunches to be fed into counter-circulating beams in the PT EDM ring. Injection into the prototype ring will be done using switching magnets distributing the beams into clockwise $(\mathrm{CW})$ or counter-clockwise $(\mathrm{CCW})$ direction as sketched in Fig. 1. All beam bunches are transferred with vertical polarization, either up or down.

Electric bends: The electrostatic deflectors consist of two cylindrically-shaped parallel metal plates with equal potential and opposite sign. With the zero voltage contour of electric potential defined to be the center line of the deflector, the ideal orbit of the design particle stays on the 


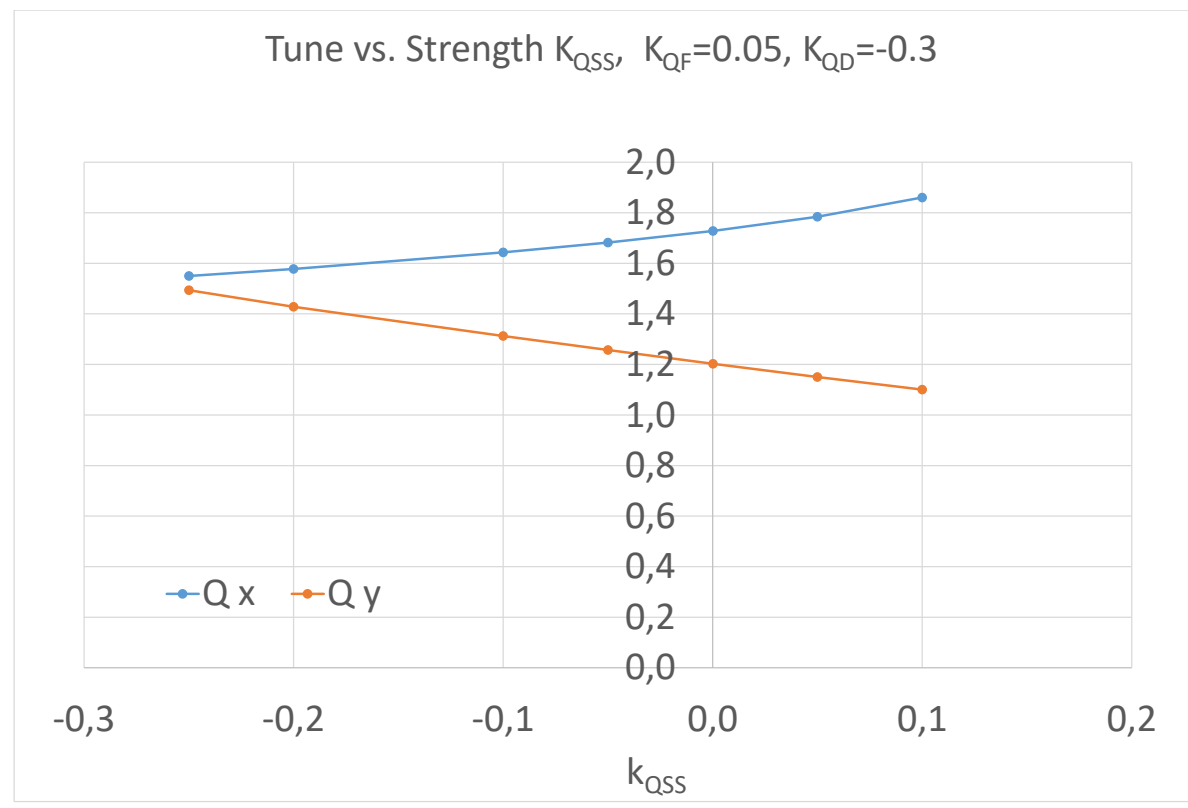

Figure 3: Additional tuning capabilities by applying the quadrupoles in the straights QSS the horizontal betatron tune $Q_{x}$ and the vertical betatron tune $Q_{y}$ are plotted versus the strength of the quadrupole family QSS. The other quadrupole families QF and QD are kept constant, as it has been marked in Fig. 2.

Table 2: Number of elements and geometry.

\begin{tabular}{lcc}
\hline & & units \\
\hline \# B-E deflectors & 8 & \\
\# arc D quads & 4 & \\
\# arc F quads & 8 & \\
\# straigh quads & 4 & \\
\hline quad length & 0.400 & $\mathrm{~m}$ \\
straight length & 8.000 & $\mathrm{~m}$ \\
bending radius & 8.861 & $\mathrm{~m}$ \\
electric plate length & 6.959 & $\mathrm{~m}$ \\
arc length (45 ) & 15.718 & $\mathrm{~m}$ \\
circumference total & 100.473 & $\mathrm{~m}$ \\
\hline
\end{tabular}

Table 3: Parameter of bend elements.

\begin{tabular}{lcc}
\hline & & units \\
\hline Electric & & \\
gap between plates & 60 & $\mathrm{~mm}$ \\
plate length & 6.959 & $\mathrm{~m}$ \\
total bending length & 55.673 & $\mathrm{~m}$ \\
total straight length & 44.800 & $\mathrm{~m}$ \\
bend angle per unit & $45^{\circ}$ & $\mathrm{m}$ \\
\hline Magnetic & & \\
magnetic field & 0.040 & $\mathrm{~T}$ \\
current density & 5.000 & $\mathrm{~A} / \mathrm{mm}^{2}$ \\
windings/element & 60 & \\
\hline
\end{tabular}

center line. The electrical potential vanishes on the center line of the bends, as well as in drift sections well outside the bends. Therefore, the electric potential vanishes everywhere on the ideal particle orbit. With the electric potential seen by the ideal particle continuous at the entrance and exit of the deflector, its total momentum is constant everywhere (even through the RF cavity). The designed ring lattice requires electric gradients in the range from 5 to $10 \mathrm{MV} / \mathrm{m}$. This is more than the standard values for most accelerator deflectors separated by a few centimeters. Assuming $60 \mathrm{~mm}$ distance between the plates, to achieve such high electric fields we have to use high voltage 


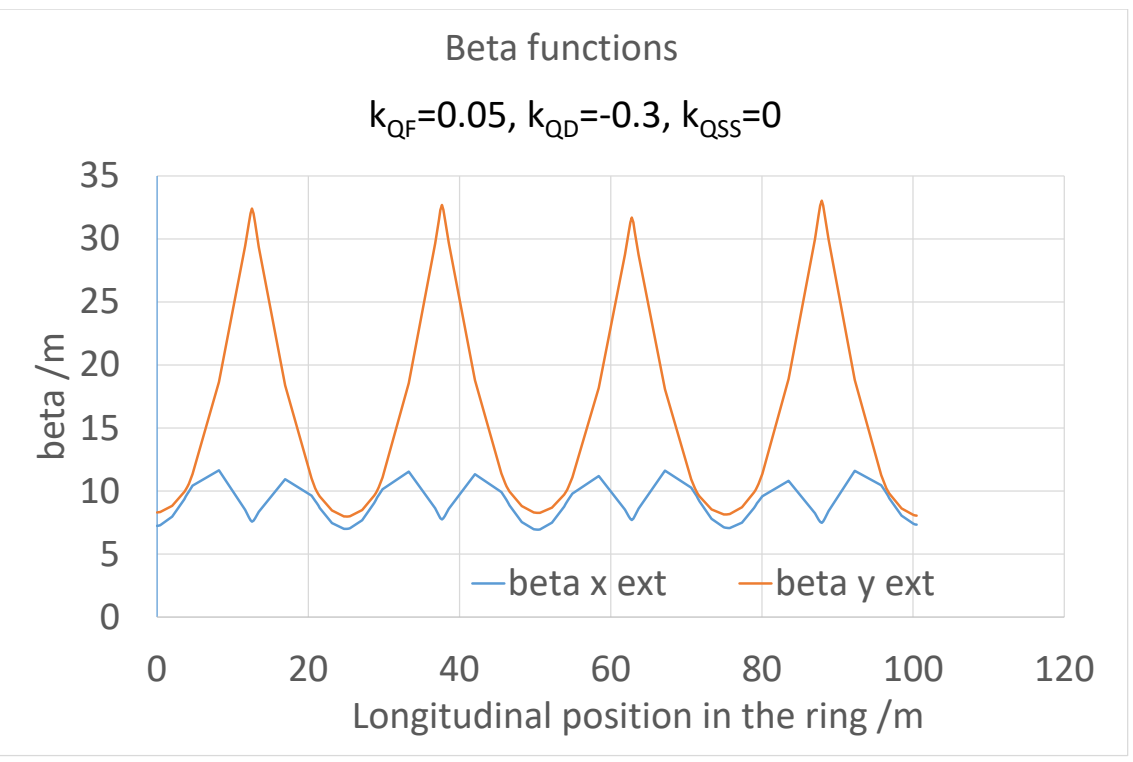

Figure 4: Beta functions for a typical working point as marked in Fig. 2: $k_{Q F}=0.05, k_{Q D}=0.3, k_{Q S S 0}=0$, with the corresponding betatron tunes of $Q_{x}=1.73$ and $Q_{y}=1.20$.

power supplies. At present, two $200 \mathrm{kV}$ power converters have been ordered for testing deflector prototypes. The field emission, field breakdown, dark current, electrode surface and conditioning will be studied using two flat electrostatic deflector plates, mounted on the movable support with the possibility of changing the separation from 20 to $120 \mathrm{~mm}$. The residual ripple of power converters is expected to be in the order of $\pm 10^{-5}$ at a maximum of $200 \mathrm{kV}$. This will lead to particle displacement on the order of millimeters. A smaller ripple or stability control of the system will be a dedicated task for investigations planned at the test ring facility.

Magnetic bends: The experiments require periodic reversals of the magnetic bending field to use symmetry to suppress systematic deviations. The reversal of the magnetic field should be done with best possible reproducibility. This is why the magnetic field production will be iron-free [9].

\section{Conclusion}

The reference energy for the PT EDM ring will be $45 \mathrm{MeV}$ protons for frozen spin experiments using combined magnetic and electric bending and $30 \mathrm{MeV}$ protons for the pure electric bending and simultaneous clockwise and counter-clockwise mode of operation. Performing the first direct, precision measurement of the proton EDM strong physics motivation requires frozen spin experiments using protons, deuterons, and eventually electrons. Further studies have to be carried out to prove, that the required beam lifetime and spin-coherence time can be reached. Handling systematic error signals remains to be the main challenge for a charged-particle EDM ring. In addition, the ability to store a sufficient number of polarized protons $\left(10^{10}\right.$ particles $)$ in a phase space of $10 \mathrm{~mm}$ mrad has to be demonstrated in a necessarily predominantly electric storage ring. Up to now, all existing relativistic rings are magnetic systems. 
The stepwise approach addressed this by the construction and operation of a small-scale prototype ring before designing the ultimate proton all-electric ring for the highest EDM sensitivity.

\section{Acknowledgements}

This work has been performed in the framework of the JEDI and CPEDM collaboration. The authors wish to acknowledge the support by the European Research Council via the ERC Advanced Grant "srEDM" (No. 694340).

\section{References}

[1] J. M. Pendlebury et al. Phys. Rev. D 92, 092003. (2015).

[2] F.J.M. Farley et al., Phys. Rev. Lett. 93, 052001 (2004); hep-ex/0307006.

[3] Storage Ring EDM Collaboration, A Proposal to Measure the Proton Electric Dipole Moment with $10^{29} \mathrm{e}-\mathrm{cm}$ Sensitivity, Brookhaven National Laboratory, October 2011.

[4] H. Stroeher for the CPEDM Collaboration, Feasibility Study for an EDM Storage Ring, scientific input submitted to ESPP (The European Strategy for Particle Physics).

[5] A. Lehrach, B. Lorentz, W. Morse, N. Nikolaev, F. Rathmann, Precursor Experiments to Search for Permanent Electric Dipole Moments (EDMs) of Protons and Deuterons at COSY, Proc. of Advanced XIV Workshop on High Energy Spin Physics 2012, Dubna, Russia.

[6] M. Berz, Computational Aspects of Design and Simulation: COSY Infinity, Nucl. Inst. Meth. A 298, 473 (1990), see also https://www.bt.pa.msu.edu/index_cosy.htm.

[7] D. Sagan, The Bmad Reference Manual, see https://www.classe.cornell.edu/bmad/manual.html.

[8] M. Rosenthal, A. Lehrach, Spin Tracking Simulations towards EDM Measurements at COSY, Proc. of the 6th International Particle Accelerator Conference (IPAC'15), Richmond, Virginia, USA, p. 3467 (2015), see http://accelconf.web.cern.ch/AccelConf/IPAC2015/papers/thpf032.pdf.

[9] H. Soltner (Forschungszentrum Jülich), private communication. 